\title{
CONGRUENCE LATTICES OF LOCALLY FINITE ALGEBRAS
}

\author{
KEITH A. KEARNES
}

\begin{abstract}
It is shown that there exist algebraic lattices that cannot be represented as the congruence lattice of a locally finite algebra.
\end{abstract}

\section{INTRODUCTION}

Recently, James Schmerl asked me if every finite lattice is isomorphic to the congruence lattice of a locally finite algebra. Noting that it is still unknown whether every finite lattice is isomorphic to the congruence lattice of a finite algebra, he reformulated the question as: Are there any finite lattices which are known to be isomorphic to the congruence lattice of an infinite locally finite algebra but not yet known to be isomorphic to the congruence lattice of a finite algebra?

I don't know the answer to either form of Schmerl's question, but have found a result of the opposite type: there exist finite lattices that are representable as the congruence lattice of a finite algebra, but not representable as the congruence lattice of an infinite locally finite algebra. Moreover, there exist algebraic lattices that are not isomorphic to the congruence lattice of any locally finite algebra at all.

To represent an algebraic lattice as the congruence lattice of an algebra, there is a lower bound on the cardinality of the representing algebra that must be satisfied. Namely, if $\mathbf{L} \cong \mathbf{C o n}(\mathbf{A})$, then $A$ must be large enough so that the lattice of all equivalence relations on $A$ contains a complete 0,1 -sublattice isomorphic to $\mathbf{L}$. Conversely, if $A$ is at least this large, and is infinite, then it is shown in Remark 2.8 that $\mathbf{L} \cong \mathbf{C o n}(\mathbf{A})$ for some algebra with universe $A$. The main purpose of this paper is to prove the existence of upper bounds on the cardinality of the representing algebra in some cases where the algebra is assumed to be locally finite. When the upper bound on cardinality is incompatible with the aforementioned lower bound, a nonrepresentability result is obtained.

\section{Nonrepresentable Lattices}

A congruence $\alpha$ on an algebra $\mathbf{A}$ is abelian if

$$
s(\mathbf{a}, \mathbf{c})=s(\mathbf{a}, \mathbf{d}) \Leftrightarrow s(\mathbf{b}, \mathbf{c})=s(\mathbf{b}, \mathbf{d})
$$

whenever $s(\mathbf{x}, \mathbf{y})$ is an $(m+n)$-ary term operation of $\mathbf{A}, \mathbf{a}, \mathbf{b}$ are $m$-tuples satisfying $\left(a_{i}, b_{i}\right) \in \alpha$ for all $i$, and $\mathbf{c}, \mathbf{d}$ are $n$-tuples satisfying $\left(c_{j}, d_{j}\right) \in \alpha$ for all $j$. This may 
be reformulated in the following way. Call $n$-ary polynomials $s_{\mathbf{a}}(\mathbf{y}):=s(\mathbf{a}, \mathbf{y})$ and $s_{\mathbf{b}}(\mathbf{y}):=s(\mathbf{b}, \mathbf{y}) \alpha$-twins if they are obtained from the same term operation $s(\mathbf{x}, \mathbf{y})$ by substituting $\alpha$-related tuples $\mathbf{a}$ and $\mathbf{b}$ for $\mathbf{x}$ respectively. Now let $N_{j}=c_{j} / \alpha=d_{j} / \alpha$ be the $\alpha$-class of $c_{j}$ for each $j$. The bi-implication (2.1) merely asserts that the two functions

$$
s_{\mathbf{a}}, s_{\mathbf{b}}: N_{1} \times \cdots \times N_{n} \rightarrow A
$$

have the same kernel. Thus, $\alpha$ is abelian if any pair of $\alpha$-twins have the same kernel when restricted to a product of $\alpha$-classes. At first, this sounds like a strange way to define abelianness, but this definition agrees with the abelianness concept from group theory and generalizes it in a useful way. (Confer $[1,3]$.)

Theorem 2.1 of [4] proves that if $\mathbf{A}$ is a finite algebra, $\mu$ is a minimal abelian congruence on $\mathbf{A}$, and $\mathbf{B}$ is a maximal proper subalgebra of $\mathbf{A}$, then $\mathbf{B}$ is either a union of $\mu$-classes or is contained in a $\mu$-transversal (which is a set containing exactly one element from every $\mu$-class). Equivalently, if a subset $X \subseteq A$ properly contains a $\mu$-transversal, then $X$ generates $\mathbf{A}$. Theorem 2.1 below partially extends this result to locally finite algebras. Namely, it proves that if $\mathbf{A}$ is a locally finite algebra and $\mu$ is a minimal abelian congruence of finite index, then any subset $X \subseteq A$ that properly contains a $\mu$-transversal generates $\mathbf{A}$. Of course, if $\mu$ has finite index, then there is a finite subset $X_{0} \subseteq A$ that properly contains a $\mu$-transversal. If $\mathbf{A}$ is generated by this finite subset, then by local finiteness $\mathbf{A}$ is finite. Conversely, if $\mathbf{A}$ is finite, then the desired result is just Theorem 2.1 of [4]. Thus, the extension to locally finite algebras should be worded as:

Theorem 2.1. Let $\mathbf{A}$ be a locally finite algebra. If $\mathbf{A}$ has a minimal abelian congruence of finite index, then $\mathbf{A}$ is finite.

Note that this result implies the nontrivial and new fact that any locally finite, abelian, simple algebra is finite. See Corollary 2.3 for a generalization. For the necessity of assuming abelianness, see Example 2.16 .

Proof. Suppose that $T$ is a $\mu$-transversal, that $X_{0}$ is a finite subset of $A$ properly containing $T$, and $\mathbf{B}$ is the subalgebra of $\mathbf{A}$ that is generated by $X_{0}$. It will be shown that $\mathbf{B}=\mathbf{A}$. Since $\mathbf{A}$ is locally finite and $\mathbf{B}$ is finitely generated, this forces $\mathbf{A}$ to be finite.

Since $T$ is a $\mu$-transversal, each element $a \in A$ is $\mu$-related to a uniquely determined element $\bar{a} \in T$. Equivalently, there is a uniquely determined function $A \rightarrow T: a \mapsto$ $\bar{a}$ for which $(a, \bar{a}) \in \mu$. It is possible to extend this notation from constants to polynomials by writing $\bar{p}$ for some (any) $\mu$-twin of $p$ whose parameters lie in $T$. More explicitly, if $p$ is a polynomial of $\mathbf{A}$, and $p(\mathbf{y})=s(\mathbf{a}, \mathbf{y})$ for some term operation $s(\mathbf{x}, \mathbf{y})$ and some tuple a of elements of $A$, then $s(\overline{\mathbf{a}}, \mathbf{y})$ is (one instance of) a polynomial that may be denoted $\bar{p}(\mathbf{y})$. 
Suppose that $N_{1} \times \cdots \times N_{n}$ is a product of $n \mu$-classes of $\mathbf{A}$. Then

$$
V:=B^{n} \cap\left(N_{1} \times \cdots \times N_{n}\right)=\left(B \cap N_{1}\right) \times \cdots \times\left(B \cap N_{n}\right)
$$

is a product of $\left.n \mu\right|_{\mathbf{B}^{-}}$-classes of $\mathbf{B}$. Let $\mathcal{P}_{n}$ be the collection of subsets of $A$ of the form $p(V)$ where $V$ is as in $(2.2), p(\mathbf{y})$ is an $n$-ary polynomial of $\mathbf{A}$. Let $\mathcal{P}=\bigcup_{n<\omega} \mathcal{P}_{n}$. Order $\mathcal{P}$ by inclusion.

Let $W=p(V) \in \mathcal{P}$ where $V$ is of the form described in $(2.2), p(\mathbf{y})=s(\mathbf{a}, \mathbf{y})$ for some term operation $s(\mathbf{x}, \mathbf{y})$, and $\bar{p}$ be a $\mu$-twin of $p$ whose parameters lie in $T \subseteq B$. Since $\mu$ is abelian, $p$ and $\bar{p}$ have the same kernel when restricted to any (subset of a) product of $\mu$-classes of $\mathbf{A}$, hence $p$ and $\bar{p}$ have the same kernel when restricted to $V$. This shows that $|p(V)|=|\bar{p}(V)|$. But $V \subseteq B^{n}$ and the sequence of parameters of $\bar{p}$ lie in $T \subseteq B$. Thus $\bar{p}(V) \subseteq s\left(B^{m}, B^{n}\right) \subseteq B$, since $s$ is a term operation and $\mathbf{B}$ is a subalgebra. It follows that $|W|=|p(V)|=|\bar{p}(V)| \leq|B|$. Since $W \in \mathcal{P}$ was arbitrary, no chain in $\mathcal{P}$ can be longer than $|B|$. In particular, any $W \in \mathcal{P}$ is contained in set $M \in \mathcal{P}$ that is maximal under inclusion.

The purpose of this paragraph is to show that the maximal elements of $\mathcal{P}$ are subsets of $B$. The idea to do this comes from [6]. Let $M \in \mathcal{P}$ be a maximal element. By the definition of $\mathcal{P}, M=p(V)$ for some set $V$ of the form described in (2.2) and some polynomial $p(\mathbf{y})=s(\mathbf{a}, \mathbf{y})=s\left(a_{1}, \ldots, a_{m}, \mathbf{y}\right)$ of $\mathbf{A}$. Since $\mathbf{B}$ properly contains a $\mu$-transversal, there is a pair $\left.(u, v) \in \mu\right|_{\mathbf{B}}$ with $u \neq v$. Let $N$ denote the $\mu$-class of $u$, and let $U=B \cap N$ denote the $\left.\mu\right|_{\mathbf{B}}$-class of $u$. Since $\left(a_{m}, \bar{a}_{m}\right) \in \mu=\operatorname{Cg}^{\mathbf{A}}(u, v)$ there is a sequence $a_{m}=w_{1}, w_{2}, \ldots, w_{k+1}=\bar{a}_{m}$ where for each $i$ there is a unary polynomial $r_{i}$ of $\mathbf{A}$ such that $\left\{w_{i}, w_{i+1}\right\}=\left\{r_{i}(u), r_{i}(v)\right\}$. The sets $M_{i}:=s\left(a_{1}, \ldots, a_{m-1}, w_{i}, V\right)$ all belong to $\mathcal{P}$ since they are polynomial images of $V$. The sets $M_{i}$ all have the same size, since for any $i$ and $j$ the $\mu$-twins $s\left(a_{1}, \ldots, a_{m-1}, w_{i}, \mathbf{y}\right)$ and $s\left(a_{1}, \ldots, a_{m-1}, w_{j}, \mathbf{y}\right)$ have the same kernel when restricted to $V . M_{1}=s\left(a_{1}, \ldots, a_{m-1}, w_{1}, V\right)=p(V)=M$ is maximal in $\mathcal{P}$. Moreover, for each $i$ the set $M_{i} \cup M_{i+1}$ is contained in a set in $\mathcal{P}$, namely the set $s\left(a_{1}, \ldots, a_{m-1}, r_{i}(U), V\right)$. That this belongs to $\mathcal{P}$ follows from the facts that $s\left(a_{1}, \ldots, a_{m-1}, r\left(y_{0}\right), \mathbf{y}\right)$ is a polynomial of $\mathbf{A}$ and $U \times V$ is of the form described in (2.2). That it contains both $M_{i}$ and $M_{i+1}$ follows from the fact that $\left\{w_{i}, w_{i+1}\right\}=\left\{r_{i}(u), r_{i}(v)\right\} \subseteq r_{i}(U)$. Altogether, it has been shown that $M_{1}, \ldots, M_{k}$ are sets in $\mathcal{P}$ of the same size, that $M_{1}=M$ is maximal under inclusion in $\mathcal{P}$, and that $M_{i} \cup M_{i+1}$ is contained in a subset of $\mathcal{P}$ for each $i$. By induction, $M_{1}=M_{2}=\cdots=M_{k}$. Thus, if $M=s\left(a_{1}, \ldots, a_{m}, V\right)$ is maximal in $\mathcal{P}$, then changing $a_{i}$ to $\bar{a}_{i}$ one by one does not change $M$. But then $M=s\left(\bar{a}_{1}, \ldots, \bar{a}_{m}, V\right) \subseteq B$, since $\bar{a}_{i} \in B$ for each $i$, $V \subseteq B^{n}, s$ is a term operation, and $\mathbf{B}$ is a subalgebra.

It was shown in the previous paragraph that the maximal elements of $\mathcal{P}$ are subsets of $B$. But then all elements of $\mathcal{P}$ are subsets of $B$, since $\mathcal{P}$ is ordered by inclusion. In particular, the minimal elements of $\mathcal{P}$, which are the sets of the form $\{a\}, a \in A$, are subsets of $B$. This proves that $A \subseteq B$, so $\mathbf{A}=\mathbf{B}$. 
It will be necessary to apply Theorem 2.1 in the situation where $\mu$ is known to be locally solvable rather than abelian, so recall from Chapter 7 of [3] the meaning of this concept. A congruence $\alpha$ on an algebra $\mathbf{A}$ is solvable if there is a finite chain of congruences $0=\alpha_{0} \leq \cdots \leq \alpha_{n}=\alpha$ such that $\alpha_{i+1} / \alpha_{i}$ is an abelian congruence of $\mathbf{A} / \alpha_{i}$. A congruence $\alpha$ on $\mathbf{A}$ is locally solvable if its restriction to any finitely generated subalgebra is solvable.

Lemma 2.2. If $\mu$ is a minimal congruence on $\mathbf{A}$, then $\mu$ is abelian if and only if it is locally solvable.

Proof. It follows easily from the definitions that any abelian congruence is locally solvable.

Arguing the contrapositive of the converse implication, suppose that $\mu$ is a minimal nonabelian congruence on $\mathbf{A}$. There exist a term $s(\mathbf{x}, \mathbf{y})$ and elements such that

$$
s(\mathbf{a}, \mathbf{c})=s(\mathbf{a}, \mathbf{d}) \quad \text { and } \quad u=s(\mathbf{b}, \mathbf{c}) \neq s(\mathbf{b}, \mathbf{d})=v,
$$

where $\left(a_{i}, b_{i}\right) \in \mu$ and $\left(c_{j}, d_{j}\right) \in \mu$ for all $i$ and $j$. Let $\mathbf{B}$ be a finitely generated subalgebra of $\mathbf{A}$ containing the finite set

$$
Y=\left\{a_{i}, b_{i}\right\}_{1 \leq i \leq m} \cup\left\{c_{j}, d_{j}\right\}_{1 \leq j \leq n} \cup\{u, v\},
$$

and enough other elements so that any two distinct $\mu$-related elements of $Y$ generate the same congruence $\nu$ of $\mathbf{B}$. This is possible since $\mu$ is a minimal congruence of $\mathbf{A}$. Using the commutator defined in Chapter 3 of [3], the implication in (2.3) implies that $(u, v) \in[\nu, \nu]$. But $[\nu, \nu] \leq \nu=\mathrm{Cg}^{\mathbf{B}}(u, v)$, so $[\nu, \nu]=\nu$. Since $0_{\mathbf{B}}<\nu=\left.[\nu, \nu] \subseteq \mu\right|_{\mathbf{B}}$, it follows that $\mu$ restricts to a nonsolvable congruence on $\mathbf{B}$, so $\mu$ is not a locally solvable congruence of $\mathbf{A}$.

An algebra is locally solvable if its largest congruence is. Congruences $\alpha$ and $\beta$ are locally solvably related, written $\alpha \stackrel{s}{\sim} \beta$, if $(\alpha \vee \beta) /(\alpha \wedge \beta)$ is a locally solvable congruence on $\mathbf{A} /(\alpha \wedge \beta)$. It is shown in Theorem 7.7 of [3] that when $\mathbf{A}$ is locally finite the relation $\stackrel{s}{\sim}$ is a complete congruence on $\operatorname{Con}(\mathbf{A})$, and $\operatorname{Con}(\mathbf{A}) / \stackrel{s}{\sim}$ is meet semidistributive. It follows from Exercise 7.14 (2) of [3] and the fact that the local solvability relation is a congruence that when $\mathbf{A}$ is locally finite and $\delta \leq \alpha \wedge \beta$ the relation $\alpha \stackrel{s}{\sim} \beta$ in $\operatorname{Con}(\mathbf{A})$ is equivalent to $\alpha / \delta \stackrel{s}{\sim} \beta / \delta$ in $\operatorname{Con}(\mathbf{A} / \delta)$.

Corollary 2.3. If $\mathbf{A}$ is a locally finite and locally solvable algebra, and $\mathbf{C o n}(\mathbf{A})$ has a finite maximal chain, then $\mathbf{A}$ is finite.

Proof. Suppose that $0=\alpha_{0} \prec \cdots \prec \alpha_{n}=1$ is a finite maximal chain in $\operatorname{Con}(\mathbf{A})$. Since $\mathbf{A}$ is locally solvable, the relation $\stackrel{s}{\sim}$ is the largest congruence on $\operatorname{Con}(\mathbf{A})$. Hence $\alpha_{i} \stackrel{s}{\sim} \alpha_{i+1}$ for each $i$, forcing $\alpha_{i+1} / \alpha_{i}$ to be a minimal locally solvable congruence of $\mathbf{A} / \alpha_{i}$. By Lemma 2.2, this congruence is abelian. It follows from Theorem 2.1 that if $\alpha_{i+1}$ has finite index, then $\alpha_{i}$ also has finite index. By descending the chain, the least congruence 0 is of finite index, hence $\mathbf{A}$ is finite. 
Definition 2.4. Let $\mathbf{L}$ be an algebraic lattice with largest element 1 . Let $\Omega$ be the smallest complete congruence on $\mathbf{L}$ such that $\mathbf{L} / \Omega$ is meet semidistributive. The $c f$-filter of $\mathbf{L}$ is the lattice filter generated by all elements $x \in L$ such that

(i) $x \equiv 1(\bmod \Omega)$, and

(ii) the interval $[x, 1]$ has a finite maximal chain.

An element in the cf-filter of $\mathbf{L}$ is called a $c$-element.

"Cf" stands for "cofinite".

Theorem 2.5. If $\mathbf{A}$ is a locally finite algebra, then every cf-element of $\mathbf{C o n}(\mathbf{A})$ is a congruence of finite index.

Proof. As noted above, $\stackrel{\mathcal{s}}{\sim}$ is a complete congruence on $\mathbf{L}:=\operatorname{Con}(\mathbf{A})$ for which the quotient $\mathbf{L} / \stackrel{s}{\sim}$ is meet semidistributive. If $\Omega$ is the smallest congruence with these properties, then $x \Omega y \Rightarrow x \stackrel{s}{\sim} y$. Therefore, the cf-filter is generated by elements $x \in L$ for which $x \stackrel{s}{\sim} 1$ and $[x, 1]$ has a finite maximal chain. For each such element, A/ $x$ is a locally solvable algebra whose congruence lattice has a finite maximal chain. According to Corollary $2.3, \mathbf{A} / x$ is finite, hence $x$ has finite index. Since the cf-filter is generated by elements of finite index, it consists of elements of finite index.

For certain lattices $\mathbf{L}$, this theorem can be used to establish an upper bound on the size of a locally finite algebra $\mathbf{A}$ for which $\mathbf{L} \cong \mathbf{C o n}(\mathbf{A})$.

Corollary 2.6. Let $\mathbf{A}$ be a locally finite algebra. If the least element of $\mathbf{L}:=\mathbf{C o n}(\mathbf{A})$ is a cf-element, then $|A|<\omega$. If the least element of $\mathbf{L}$ is the meet of $\leq \kappa$ cf-elements of $\mathbf{L}$, for some infinite $\kappa$, then $|A| \leq 2^{\kappa}$.

Proof. The first claim is a direct consequence of Theorem 2.5. For the second claim, if the least element of $\operatorname{Con}(\mathbf{A})$ is the intersection of $\leq \kappa$ cf-elements, then from Theorem 2.5 the algebra $\mathbf{A}$ is a subdirect product of $\leq \kappa$ finite algebras. If $\kappa$ is infinite, this forces $|A| \leq \omega^{\kappa}=2^{\kappa}$.

In particular, this shows that if the least element of $\mathbf{L}$ is the meet of $\leq \kappa$ cfelements, and $|L|>2^{2^{\kappa}}$, then $\mathbf{L}$ is not representable as the congruence lattice of a locally finite algebra (since a set of size $\leq 2^{\kappa}$ supports $\leq 2^{2^{\kappa}}$ equivalence relations). More generally, if $x \in L$ is above the meet of $\kappa$ cf-elements, and the interval [ $x, 1]$ has cardinality greater than $2^{2^{\kappa}}$, then $\mathbf{L}$ is not representable as the congruence lattice of a locally finite algebra.

Next is a nonrepresentability result whose statement does not refer explicitly to cf-elements.

Corollary 2.7. Let $\mathbf{L}$ be an algebraic lattice. Suppose that

(i) L has a finite maximal chain, and

(ii) there is no complete homomorphism from $\mathbf{L}$ onto a 2-element chain. 
If $\mathbf{L} \cong \mathbf{C o n}(\mathbf{A})$ for some locally finite algebra $\mathbf{A}$, then $\mathbf{A}$ is finite. In particular, if $\mathbf{L}$ is an infinite algebraic lattice satisfying (i) and (ii), then $\mathbf{L}$ is not isomorphic to the congruence lattice of a locally finite algebra.

Proof. The proof of the corollary is accomplished by showing that if (i) and (ii) hold, then the least element of $\mathbf{L}$ is a cf-element. Then the conclusion follows from the first part of Corollary 2.6.

Let $\Omega$ be the least complete congruence on $\mathbf{L}$ such that $\mathbf{L} / \Omega$ is meet semidistributive. If $\Omega$ is not the total binary relation on $\mathbf{L}$, then $\mathbf{L} / \Omega$ is a nontrivial, complete, meet semidistributive lattice. Since $\mathbf{L}$ is algebraic, it is meet continuous. The natural map $\nu: \mathbf{L} \rightarrow \mathbf{L} / \Omega$ is complete, so $\mathbf{L} / \Omega$ is also meet continuous. Since $\mathbf{L} / \Omega$ has a finite maximal chain, it has an atom $\alpha$. The map $\varphi: \mathbf{L} / \Omega \rightarrow[0, \alpha]: x \mapsto x \wedge \alpha$ is a complete lattice homomorphism onto a 2-element chain. (That $\varphi$ preserves complete joins uses the meet semidistributivity and meet continuity of $\mathbf{L} / \Omega$.) But then $\varphi \circ \nu$ is a complete homomorphism of $\mathbf{L}$ onto a 2-element chain, contrary to (ii). Therefore $\Omega$ is the total binary relation on $\mathbf{L}$.

If 0 and 1 are the least and largest elements of $\mathbf{L}$, then $0 \equiv 1(\bmod \Omega)$ by the conclusion of the previous paragraph. Since $[0,1]=L$ has a finite maximal chain, 0 is a cf-element.

Remark 2.8. It is asserted in the introduction of this paper that if $\mathbf{L}$ is an algebraic lattice and the lattice of all equivalence relations on the infinite set $A$ contains a complete 0,1-sublattice isomorphic to $\mathbf{L}$, then there is an algebra $\mathbf{A}$ with universe $A$ such that $\mathbf{L} \cong \operatorname{Con}(\mathbf{A})$. This claim will be supported now.

If $\mathbf{L}$ is isomorphic to a complete 0,1-sublattice of $\mathbf{E q}(A)$, then the number $\kappa$ of compact elements of $\mathbf{L}$ does not exceed the number of compact elements of $\mathbf{E q}(A)$, which is $|A|$. Thus, the least possible cardinality for the universe $A$ of an infinite representing algebra for $\mathbf{L}$ is $\kappa+\omega$. The constructions in $[2,5,8]$ produce a representing algebra $\mathbf{A}$ of this size. The fact that representing algebras exist in all larger cardinalities can be proved by slightly modifying the constructions in $[2,5,8]$, or by using the generalized matrix power construction described in the next paragraph.

Let $\mathbf{A}$ be an algebra, and let $\mathbf{B}$ be a Boolean algebra with Stone space $B^{*}$. Let $\mathbf{A}[\mathbf{B}]^{*}$ be the Boolean power of $\mathbf{A}$ by $\mathbf{B}$, which is the subalgebra of the direct power $\mathbf{A}^{B^{*}}$ consisting of all continuous functions from $B^{*}$ to the discrete space $A$. Let $\mathbf{A}^{[\mathbf{B}]}$ be the expansion of $\mathbf{A}[\mathbf{B}]^{*}$ obtained by adding the following new operations:

(i) for each homeomorphism $h: B^{*} \rightarrow B^{*}$ add a unary operation $u_{h}$ defined by $u_{h}(f)=f \circ h^{-1}$, and

(ii) for each clopen set $U \subseteq B^{*}$ add a binary operation $b_{U}$ defined by $b_{U}(f, g)=$ $\left.\left.f\right|_{U} \cup g\right|_{B^{*} \backslash U}$.

A finitary relational clone on a set $A$ is an infinitary multisorted algebra whose universe is a set $\mathcal{R}$ of finitary relations on $A$, graded by arity, which contains the binary equality relation on $A$. Its operations are the binary product $(\times)$ of relations 
of possibly different arities, arbitrary intersection $(\cap)$ of relations of the same arity, unary projections $\left(p_{I}^{n}\right)$ of $n$-ary relations onto $(n-1)$-element subsets $I$ of the $n$ coordinates, unary permutations of coordinates $\left(\Pi_{\sigma}\right)$ of $n$-ary relations for all $\sigma \in S_{n}$ and all $n<\omega$, and directed unions $(\cup)$ of $n$-ary relations for each $n<\omega$. It is known that for any algebra $\mathbf{A}$ the set of finitary compatible relations of $\mathbf{A}$ forms a finitary relational clone (written $\operatorname{Rel}(\mathbf{A})$ ), and that every finitary relational clone is $\operatorname{Rel}(\mathbf{A})$ for some algebra $\mathbf{A}$ (cf. [7, 9]). The elements of $\mathbf{C o n}(\mathbf{A})$ are among the elements of the binary component of $\operatorname{Rel}(\mathbf{A})$ and the complete lattice operations of $\operatorname{Con}(\mathbf{A})$ are the restrictions to $\operatorname{Con}(\mathbf{A})$ of term operations of $\operatorname{Rel}(\mathbf{A})$, so $\operatorname{Con}(\mathbf{A})$ is a subalgebra of a reduct of $\operatorname{Rel}(\mathbf{A})$. Since $\operatorname{Con}(\mathbf{A})$ is an equationally definable subset of $\operatorname{Rel}(\mathbf{A})$, if $\operatorname{Rel}(\mathbf{A}) \cong \operatorname{Rel}(B)$ then $\operatorname{Con}(A) \cong \operatorname{Con}(B)$.

If $\rho$ is an $n$-ary compatible relation of $\mathbf{A}$, then it is a subuniverse of $\mathbf{A}^{n}$. For any Boolean algebra $\mathbf{B}$ the Boolean power $\rho[\mathbf{B}]^{*}$ is a subuniverse of $\mathbf{A}^{n}[\mathbf{B}]^{*} \cong\left(\mathbf{A}[\mathbf{B}]^{*}\right)^{n}$, hence may be viewed as a compatible $n$-ary relation of $\mathbf{A}[\mathbf{B}]^{*} . \rho[\mathbf{B}]^{*}$ is compatible with the operations $u_{h}$ and $b_{U}$ defined earlier, so $\rho[\mathbf{B}]^{*}$ may be viewed as a compatible $n$-ary relation on $\mathbf{A}^{[\mathbf{B}]}$. When viewed this way, $\rho[\mathbf{B}]^{*}$ will be denoted $\rho^{[\mathbf{B}]}$.

A congruence $\theta$ of a Boolean algebra $\mathbf{B}$ is characteristic if $\alpha(\theta)=\theta$ for every automorphism $\alpha \in \operatorname{Aut}(\mathbf{B})$. B is characteristically simple if its only characteristic congruences are $0_{\mathbf{B}}$ and $1_{\mathbf{B}}$. Ideals and filters of $\mathbf{B}$ are characteristic if they generate characteristic congruences.

Theorem 2.9. The following conditions are equivalent for a nontrivial Boolean algebra $\mathbf{B}$.

(1) For some (equivalently every) nontrivial algebra $\mathbf{A}$, the mapping $\rho \mapsto \rho^{[\mathbf{B}]}$ is an isomorphism from $\boldsymbol{\operatorname { R e l }}(\mathbf{A})$ to $\operatorname{Rel}\left(\mathbf{A}^{[\mathbf{B}]}\right)$.

(2) For some (equivalently every) nontrivial algebra $\mathbf{A}$, the mapping $\theta \mapsto \theta^{[\mathbf{B}]}$ is an isomorphism from $\mathbf{C o n}(\mathbf{A})$ to $\operatorname{Con}\left(\mathbf{A}^{[\mathbf{B}]}\right)$.

(3) $\mathbf{B}$ is characteristically simple.

Proof. By Stone duality, we may assume throughout the proof that $\mathbf{B}$ is the Boolean algebra of clopen subsets of the Stone space $B^{*}$, and that the automorphisms of $\mathbf{B}$ are those induced by homeomorphisms of $B^{*}$.

$[(1) \Rightarrow(2)]$ : If (1) holds for some nontrivial algebra $\mathbf{A}$, then (2) also holds for $\mathbf{A}$ since $\operatorname{Con}(\mathbf{A})$ is an equationally definable subalgebra of a reduct of $\operatorname{Rel}(\mathbf{A})$.

$[\neg(3) \Rightarrow \neg(2)]$ : If $F$ is a proper, nontrivial, characteristic filter of $\mathbf{B}$, then the set $C$ of ultrafilters of $\mathbf{B}$ extending $F$ is a proper, nonempty, closed subset of the Stone space $B^{*}$ that is invariant under all homeomorphisms of $B^{*}$. The binary relation $\Theta$ defined on $\mathbf{A}^{[\mathbf{B}]}$ by $(f, g) \in \Theta$ iff $\left.f\right|_{C}=\left.g\right|_{C}$ is a congruence of $\mathbf{A}^{[\mathbf{B}]}$. It cannot be of the form $\theta^{[\mathbf{B}]}$ for any $\theta \in \operatorname{Con}(\mathbf{A})$, since under the $p$-th coordinate projection homomorphism $\pi_{p}: \mathbf{A}^{[\mathbf{B}]} \rightarrow \mathbf{A}: f \mapsto f(p)$ we have $\pi_{p}\left(\theta^{[\mathbf{B}]}\right)=\theta$ for any point $p \in B^{*}$. But $\pi_{p}(\Theta)$ equals $0_{\mathbf{A}}$ when $p \in C$ and equals $1_{\mathbf{A}}$ when $p \notin C$, and $0_{\mathbf{A}} \neq 1_{\mathbf{A}}$ when $\mathbf{A}$ is nontrivial. 
$[(3) \Rightarrow(1)]$ : The verification of the fact that the mapping $\rho \mapsto \rho^{[\mathbf{B}]}$ is an injective homomorphism from $\operatorname{Rel}(\mathbf{A})$ to $\operatorname{Rel}\left(\mathbf{A}^{[\mathbf{B}]}\right)$ is routine and does not require the assumption that $\mathbf{B}$ is characteristically simple. We argue only that if $\mathbf{B}$ is characteristically simple, then the function $\operatorname{Rel}(\mathbf{A}) \rightarrow \operatorname{Rel}\left(\mathbf{A}^{[\mathbf{B}]}\right): \rho \mapsto \rho^{[\mathbf{B}]}$ is surjective.

For a clopen set $U \in B$ and a homeomorphism $h: B^{*} \rightarrow B^{*}$, call $U(h):=h(U)$ the translate of $U$ by $h$. The assumption that $\mathbf{B}$ is characteristically simple means that the characteristic ideal generated by any nonempty $U \in B$ contains the set $B$. But the characteristic ideal generated by $U$ is the ordinary ideal generated by the translates of $U$. Hence $\mathbf{B}$ is characteristically simple iff for any nonempty clopen set $U \in B$ it is possible to express $B$ as a union of finitely many translates of $U$.

Choose any compatible relation $R \in \operatorname{Rel}\left(\mathbf{A}^{[\mathbf{B}]}\right)$, and any point $p \in B^{*}$. Let $\rho=\pi_{p}(R)$. If $a \in A$, let $c_{a}: B^{*} \rightarrow A$ be the constant function with image $\{a\}$.

Claim 2.10. A tuple $\left(a_{1}, \ldots, a_{n}\right)$ of elements of $A$ belongs to $\rho$ if and only if the corresponding tuple $\left(c_{a_{1}}, \ldots, c_{a_{n}}\right)$ of constant functions in $A^{[\mathbf{B}]}$ belongs to $R$.

If $\left(c_{a_{1}}, \ldots, c_{a_{n}}\right) \in R$, then $\left(a_{1}, \ldots, a_{n}\right)=\pi_{p}\left(\left(c_{a_{1}}, \ldots, c_{a_{n}}\right)\right) \in \rho$ according to the definition of $\rho$. Conversely, if $\left(a_{1}, \ldots, a_{n}\right) \in \rho$, then there exist functions $f_{1}, \ldots, f_{n} \in A^{[\mathbf{B}]}$ such that $\left(f_{1}, \ldots, f_{n}\right) \in R$ and $\pi_{p}\left(\left(f_{1}, \ldots, f_{n}\right)\right)=\left(f_{1}(p), \ldots, f_{n}(p)\right)=\left(a_{1}, \ldots, a_{n}\right)$. Since the universe of $\mathbf{A}^{[\mathbf{B}]}$ is $A[\mathbf{B}]^{*}$, there is a clopen set $U$ containing $p$ such that $\left.f_{i}\right|_{U}$ is constant for all $i$. Thus $\left.\left(f_{1}, \ldots, f_{n}\right)\right|_{U}=\left.\left(c_{a_{1}}, \ldots, c_{a_{n}}\right)\right|_{U}$. Since $\mathbf{B}$ is characteristically simple and $U \neq \emptyset$, there is a finite sequence of translates $U\left(h_{j}\right), 1 \leq j \leq \ell$, whose union is $B$. For each $j$, the tuple $u_{h_{j}}\left(f_{1}, \ldots, f_{n}\right)=\left(f_{1} \circ h_{j}^{-1}, \ldots, f_{n} \circ h_{j}^{-1}\right)$ belongs to $R$, since $u_{h_{j}}$ is a term operation, and it agrees with $u_{h_{j}}\left(c_{a_{1}}, \ldots, c_{a_{n}}\right)=\left(c_{a_{1}}, \ldots, c_{a_{n}}\right)$ on $U\left(h_{j}\right)$.

We prove by induction on $k$ that $R$ contains a tuple that agrees with $\left(c_{a_{1}}, \ldots, c_{a_{n}}\right)$ on $U\left(h_{1}\right) \cup \cdots \cup U\left(h_{k}\right)$. The base case was established in the preceding paragraph. Let $V$ equal the clopen set $U\left(h_{1}\right) \cup \cdots \cup U\left(h_{k}\right)$. If $\left(g_{1}, \ldots, g_{n}\right) \in R$ agrees with $\left(c_{a_{1}}, \ldots, c_{a_{n}}\right)$ on $V$, then since $u_{h_{k+1}}\left(f_{1}, \ldots, f_{n}\right) \in R$ agrees with $\left(c_{a_{1}}, \ldots, c_{a_{n}}\right)$ on $U\left(h_{k+1}\right)$, it follows that

$$
b_{V}\left(\left(g_{1}, \ldots, g_{n}\right), u_{h_{k+1}}\left(f_{1}, \ldots, f_{n}\right)\right) \in R
$$

agrees with $\left(g_{1}, \ldots, g_{n}\right)$ on $V$ and with $\left(f_{1}, \ldots, f_{n}\right)$ on $B^{*} \backslash V$. Thus, it is a tuple in $R$ that agrees with $\left(c_{a_{1}}, \ldots, c_{a_{n}}\right)$ on $V \cup U\left(h_{k+1}\right)$, which is what was to be proved. By induction, $R$ contains a tuple that agrees with $\left(c_{a_{1}}, \ldots, c_{a_{n}}\right)$ on $U\left(h_{1}\right) \cup \cdots \cup U\left(h_{\ell}\right)=$ $B^{*}$, hence $\left(c_{a_{1}}, \ldots, c_{a_{n}}\right) \in R$.

Claim 2.11. $\pi_{q}(R)=\rho$ for every $q \in B^{*}$.

According to Claim 2.10, if $C$ is the set of tuples in $R$ consisting of constant functions, then $\pi_{p}(R)=\pi_{p}(C)$ for a arbitrarily chosen point $p \in B^{*}$. Thus $\pi_{p}(R)$ is independent of $p$.

Claim 2.12. $R=\rho^{[\mathrm{B}]}$. 
From Claim 2.11, $R$ is contained in the restriction of full power $\rho^{B^{*}}$ to $A^{[\mathbf{B}]}$. But this restiction is just $\rho^{[\mathbf{B}]}$, so $R \subseteq \rho^{[\mathbf{B}]}$. Now choose any tuple $\left(f_{1}, \ldots, f_{n}\right) \in \rho^{[\mathbf{B}]}$. There exists a partition $U_{1} \cup \cdots \cup U_{k}$ of $B^{*}$ into clopen sets such that each $f_{i}$ is constant on each $U_{j}$; say $\left.\left(f_{1}, \ldots, f_{n}\right)\right|_{U_{j}}=\left.\left(c_{1}^{j}, \ldots, c_{n}^{j}\right)\right|_{U_{j}}$ for constant functions $c_{i}^{j}$, $1 \leq j \leq k$. For $p \in U_{j}$ we have

$$
\left(c_{1}^{j}(p), \ldots, c_{n}^{j}(p)\right)=\left(f_{1}(p), \ldots, f_{n}(p)\right) \in \rho,
$$

so $\left(c_{1}^{j}, \ldots, c_{n}^{j}\right) \in R$ for all $j$.

We argue by induction on $j$ that $R$ contains a tuple that agrees with $\left(f_{1}, \ldots, f_{n}\right)$ on $U_{1} \cup \cdots \cup U_{j}$. The tuple $\left(c_{1}^{1}, \ldots, c_{n}^{1}\right) \in R$ proves this when $j=1$. Suppose that $\left(g_{1}, \ldots, g_{n}\right) \in R$ agrees with $\left(f_{1}, \ldots, f_{n}\right)$ on $W:=U_{1} \cup \cdots \cup U_{j}$. Then

$$
b_{W}\left(\left(g_{1}, \ldots, g_{n}\right),\left(c_{1}^{j+1}, \ldots, c_{n}^{j+1}\right)\right) \in R
$$

and this tuple agrees with $\left(f_{1}, \ldots, f_{n}\right)$ on $U_{1} \cup \cdots \cup U_{j} \cup U_{j+1}$. By induction, $\left(f_{1}, \ldots, f_{n}\right) \in R$. This completes the proof that the mapping $\rho \mapsto \rho^{[\mathbf{B}]}$ is surjective for every $\mathbf{A}$.

Free Boolean algebras are characteristically simple. Therefore if $\mathbf{B}$ is a free Boolean algebra of infinite rank $\lambda \geq|A|$, then $\mathbf{A}^{[\mathbf{B}]}$ has cardinality equal to $\left|A^{[\mathbf{B}]}\right|=\left|A[\mathbf{B}]^{*}\right|=$ $|A| \cdot|B|=\max (|A|, \lambda)=\lambda$, and the map $\theta \mapsto \theta^{[\mathbf{B}]}$ is an isomorphism of $\mathbf{C o n}(\mathbf{A})$ onto $\operatorname{Con}\left(\mathbf{A}^{[\mathbf{B}]}\right)$. This shows that if $\mathbf{L}$ is representable as $\operatorname{Con}(\mathbf{A})$ for some algebra $\mathbf{A}$, then $\mathbf{L}$ is representable as the congruence lattice of an algebra of size $\lambda$ for every infinite $\lambda \geq|A|$.

Example 2.13. Let $X$ be an infinite set of cardinality $\kappa$, and let $\mathbf{L}=\mathbf{E q}(X)$ be the lattice of all equivalence relations on $X$. If $\mathbf{X}=\langle X$; $\emptyset\rangle$, then $\mathbf{L}=\mathbf{C o n}(\mathbf{X})$, so $\mathbf{L}$ is representable as the congruence lattice of a locally finite algebra of size $\kappa$. By Remark 2.8, if $\mathbf{L}$ is representable in cardinality $\kappa$, then it is representable in all larger cardinalities. However, the generalized matrix power construction of Remark 2.8 usually does not produce locally finite algebras. $\left(\mathbf{A}^{[\mathbf{B}]}\right.$ is locally finite if and only if $\mathbf{A}$ is locally finite and $\mathbf{B}$ is finite.) Here it will be shown that $\mathbf{L}$ has infinitely many different representations as the congruence lattice of a locally finite algebra of cardinality $\kappa$, but no representation as the congruence lattice of a locally finite algebra of any other cardinality.

$\mathbf{L}$ has infinitely many different representations in cardinality $\kappa$ simply because the ordinary matrix power $\mathbf{X}^{[k]}$ has a congruence lattice isomorphic to $\mathbf{L}=\mathbf{C o n}(\mathbf{X})$ for each $k$. The cardinality of $\mathbf{X}^{[k]}$ is $\left|X^{k}\right|=|X|=\kappa$. Such representations are "different" for different values of $k$ because maximal congruences on $\mathbf{X}^{[k]}$ have index $2^{k}$, and this changes as $k$ does.

Since the lattice of equivalence relations on a $\kappa$-element set has $\kappa$ compact elements when $\kappa \geq \omega$, it is clear that $\mathbf{L}$ cannot be represented as a congruence lattice of any algebra of cardinality less than $\kappa$. In this paragraph it will be shown that $\mathbf{L}$ cannot be 
represented as a congruence lattice of a locally finite algebra in cardinalities greater $\kappa$. Indeed, suppose that $\varphi: \mathbf{L} \rightarrow \operatorname{Con}(\mathbf{A})$ is an isomorphism, where $\mathbf{A}$ is a locally finite algebra. It is easy to see that the elements $\theta \in L=\operatorname{Eq}(X)$ that are of finite index are cf-elements, and that the compact elements of $L$ are the equivalence relations on $X$ with finitely many nonsingleton classes, each one finite. Therefore, $\mathbf{L}$ has $\kappa$ compact elements, and if $\alpha \in L$ is compact, then $\alpha$ has a lattice-theoretic complement that is a cf-element. Using the isomorphism $\varphi$, we obtain that $\operatorname{Con}(\mathbf{A})$ has $\kappa$-many compact elements, and each one has a lattice-theoretic complement that is a cf-element. Since $\mathbf{A}$ is locally finite, the cf-elements have finite index. If a congruence $\alpha$ on $\mathbf{A}$ has a cf-element $\theta$ as a complement, and the index of $\theta$ is $m$, then the classes of $\alpha$ have size $\leq m$. Therefore, if $a \in A$ is fixed and $Y:=\{a / \alpha \mid \alpha \in \operatorname{Con}(\mathbf{A})$ compact $\}$, then $Y$ consists of at most $\kappa$-many finite subsets of $A$, so $|\bigcup Y| \leq \kappa$. But if $b \in A$, then $b \in a / \operatorname{Cg}(a, b)$ and $a / \operatorname{Cg}(a, b) \in Y$. Therefore $A=\bigcup Y$ has size at most $\kappa$.

Example 2.14. Let $V$ be a vector space of dimension greater than 1. Every subspace of finite codimension is a cf-element in the lattice $\operatorname{Sub}(V)$ of subspaces of $V$. If $\operatorname{Sub}(V) \cong \operatorname{Con}(\mathbf{A})$ for some locally finite algebra $\mathbf{A}$, then it follows from Theorem 2.5 that $V / U$ is finite when $U$ has finite codimension. This forces $V$ to be a vector space over a finite field, implying that $V$ itself is locally finite. In other words, if the congruence lattice of a vector space of dimension $\geq 1$ is representable as the congruence lattice of a locally finite algebra, then the vector space itself must be locally finite. Moreover, by arguments mirroring those of Example 2.13, the vector space and the representing algebra must have the same size.

Example 2.15. Let $G$ be a group, and let $\mathbf{L}=\operatorname{Sub}(G)$ be the lattice of subgroups of $G$. Then $\mathbf{L}$ is isomorphic to the congruence lattice of $G$ considered as a $G$-set over itself. Such an algebra is never simultaneously locally finite and infinite, and it seems to happen frequently that the lattice $\mathbf{L}$ is not representable as the congruence lattice of any algebra that is simultaneously locally finite and infinite.

For example, if $H$ is a nontrivial finite group and $G=H \times H$, then $\operatorname{Sub}(G)$ contains elements $H \times\{1\},\{1\} \times H$, and the diagonal subgroup $D=\{(h, h) \mid h \in H\}$. These three subgroups pairwise join to $G$ and pairwise meet to $\{1\}$. This is enough to show that $\operatorname{Sub}(G)$ has no homomorphism onto the 2-element chain. By Corollary 2.7, $\operatorname{Sub}(G)$ is not isomorphic to the congruence lattice of an infinite locally finite algebra.

For another example, it can be argued that if $G$ is any nontrivial finite group satisfying $[G, G]=G$, then $\operatorname{Sub}(G)$ has no homomorphism onto a 2-element chain. (For if $\varphi: \operatorname{Sub}(G) \rightarrow \mathbf{2}$, then the largest subgroup $\varphi$ maps to zero can be shown to be normal in $G$ of prime power index. Thus, if $[G, G]=G$, then there is no such normal subgroup, so there can be no such homomorphism.) By Corollary 2.7, in this situation $\operatorname{Sub}(G)$ is not isomorphic to the congruence lattice of an infinite locally finite algebra. 
Some subgroup lattices are not isomorphic to the congruence lattice of any locally finite algebra at all. For example, let $G=S O(3, \mathbb{R})$ be the special orthogonal group, viewed as the rotation group of the unit sphere in $\mathbb{R}^{3}$. For a on the unit sphere, the stabilizer $G_{\mathbf{a}}$ consists of the rotations around the axis whose direction vector is $\mathbf{a}$. If $G_{\mathbf{a}} \neq G_{\mathbf{b}}$, then $G_{\mathbf{a}} \wedge G_{\mathbf{b}}=\{1\}$ and $G_{\mathbf{a}} \vee G_{\mathbf{b}}=G$ in $\mathbf{L}$. This is enough to prove that the least complete congruence $\Omega$ such that $\mathbf{L} / \Omega$ is meet semidistributive is the total relation. Hence the cf-filter of $\mathbf{L}$ is generated by those $H \in L$ such that the interval $[H, G]$ contains a finite maximal chain. Since the interval $\left[G_{\mathbf{a}}, G\right]$ contains only three elements, namely $G_{\mathbf{a}}, G$ and the setwise stabilizer $G_{\{\mathbf{a},-\mathbf{a}\}}$ of $\{\mathbf{a},-\mathbf{a}\}$, it follows that each $G_{\mathbf{a}}$ is in the cf-filter. Since $G_{\mathbf{a}} \wedge G_{\mathbf{b}}=\{1\}$ when $G_{\mathbf{a}} \neq G_{\mathbf{b}}$, it follows that $\{1\}$ is a cf-element. By Theorem 2.5, $\mathrm{L}$ is not the congruence lattice of a locally finite algebra.

Example 2.16. It has been shown that certain finite lattices are not representable as the congruence lattice of an infinite locally finite algebra. Here is will be shown that every finite distributive lattice is the congruence lattice of a locally finite algebra of cardinality $\kappa$ for any infinite $\kappa$ (and for infinitely many finite $\kappa$ ).

Let $\mathbf{D}$ be a finite distributive lattice, and let $\mathbf{B}$ be a Boolean lattice of cardinality $\kappa$ that contains $\mathbf{D}$ as a 0,1 -sublattice. For each $x \in B$, let $c(x)$ be the least element of the sublattice $\mathbf{D}$ that lies above $x$. Then $c: B \rightarrow B$ is an increasing join homomorphism whose fixed points are the elements of $D$. Let $\mathbf{A}=\langle B ; \vee, \wedge, c\rangle$.

A is locally finite, since if $\mathbf{C}$ is a subalgebra generated by a finite subset $X_{0} \subseteq B$, then $\mathbf{C}$ is contained in the sublattice of $\mathbf{B}$ generated by the finite set $X_{0} \cup D$. Since $\mathbf{B}$ is a locally finite lattice, $\mathbf{C}$ is finite.

Any congruence on $\mathbf{A}$ is a congruence on the Boolean lattice $\mathbf{B}$, hence is uniquely determined by the ideal $I$ of elements congruent to 0 . For the congruence to be compatible with $c$ also, it is necessary to have $c(I) \subseteq I$, which means that $I$ must be a principal ideal generated by an element of $D$. Conversely, if $I$ is a principal ideal generated by an element $d \in D$, then the lattice congruence corresponding to $I$ is $\operatorname{Cg}^{\mathbf{B}}(0, d)=\left\{(x, y) \in B^{2} \mid x \vee d=y \vee d\right\}$. This is the kernel of the lattice endomorphism $x \mapsto x \vee d$ of $\mathbf{B}$, which is readily seen to be an endomorphism of $\mathbf{A}$ also. Hence $\mathrm{Cg}^{\mathbf{B}}(0, d)$ is a congruence of $\mathbf{A}$ when $d \in D$. Altogether this shows that the congruences of $\mathbf{A}$ are those of the form $\mathrm{Cg}^{\mathbf{B}}(0, d), d \in D$. Since $\mathbf{D}$ is a sublattice, $\operatorname{Cg}(0, x) \vee \operatorname{Cg}(0, y)=\operatorname{Cg}(0, x \vee y)$ and $\operatorname{Cg}(0, x) \wedge \operatorname{Cg}(0, y)=\operatorname{Cg}(0, x \wedge y)$. This proves that $\varphi: \mathbf{D} \rightarrow \mathbf{C o n}(\mathbf{A}): x \mapsto \operatorname{Cg}(0, x)$ is an isomorphism.

\section{REFERENCES}

[1] R. Freese and R. McKenzie, Commutator Theory for Congruence Modular Varieties, London Mathematical Society Lecture Note Series, 125. Cambridge University Press, Cambridge, 1987.

[2] G. Grätzer and E. T. Schmidt, Characterizations of congruence lattices of abstract algebras, Acta Sci. Math. (Szeged) 24 (1963), 34-59. 
[3] D. Hobby and R. McKenzie, The Structure of Finite Algebras, Contemporary Mathematics, 76, American Mathematical Society, 1988.

[4] K. A. Kearnes, A Hamiltonian property for nilpotent algebras, Algebra Universalis 37 (1997), no. 4, 403-421.

[5] W. Lampe, On the congruence lattice characterization theorem, Trans. Amer. Math. Soc. 182 (1973), 43-60.

[6] E. W. Kiss, M. A. Valeriote, Abelian algebras and the Hamiltonian property, J. Pure Appl. Algebra 87 (1993), no. 1, 37-49.

[7] R. Pöschel, Concrete representation of algebraic structures and a general Galois theory, Contributions to General Algebra (Proc. Klagenfurt Conf., Klagenfurt, 1978), pp. 249-272, Heyn, Klagenfurt, 1979.

[8] P. Pudlák, A new proof of the congruence lattice representation theorem, Algebra Universalis 6 (1976), no. 3, 269-275.

[9] L. Szabó, Concrete representation of related structures of universal algebras. I, Acta Sci. Math. (Szeged) 40 (1978), no. 1-2, 175-184.

(Keith Kearnes) Department of Mathematics, University of Colorado, Boulder CO 80309, USA

E-mail address: kearnes@euclid.colorado.edu 